\title{
A hybrid fluid-kinetic neutral model based on a micro-macro decomposition in the SOLPS-ITER plasma edge code suite
}

\author{
N. Horsten* ${ }^{1}$ W. Dekeyser, ${ }^{1}$ M. Blommaert, ${ }^{1}$ G. Samaey, ${ }^{2}$ and M. Baelmans ${ }^{1}$ \\ ${ }^{1}$ Department of Mechanical Engineering, KU Leuven, Leuven, Belgium \\ ${ }^{2}$ Department of Computer Science, KU Leuven, Leuven, Belgium
}

Correspondence: *N. Horsten, Celestijnenlaan 300A, 3001 Leuven, Belgium. Email: niels.horsten@kuleuven.be

Received 16 September, 2019; Revised 6 January, 2020; Accepted 10 February, 2020

DOI 10.1002/ctpp.201900132

We present a hybrid fluid-kinetic model for the hydrogenic atoms in the plasma edge, which is implemented in SOLPS-ITER. A micro-macro decomposition of the kinetic equation leads to a fluid model with a continuity and parallel momentum equation (implemented in B2.5) coupled to a kinetic correction equation (implemented in EIRENE). We assess the hybrid model for a high recycling fixed background plasma. The hybrid approach leads to a reduction of the CPU time to obtain the same statistical error as the full kinetic Monte Carlo (MC) simulation with approximately factors $1.7,4.9$, and 1.9 for respectively the particle, parallel momentum, and electron energy source. However, there is a CPU time increase for the ion energy source. By comparing the results with our in-house plasma edge code, we conclude that the hybrid performance can be improved by adapting some default MC features in EIRENE.

Keywords: Fluid approximations; Kinetic model; Neutrals; Plasma edge modeling

\section{Introduction}

There are two main approaches to model the neutral particles (atoms and molecules) in the plasma edge. On the one hand, they can be treated kinetically with a Monte Carlo (MC) simulation, with, e.g., the EIRENE code 1. This facilitates the incorporation of multiple (kinds of) species and microscopic processes. The kinetic MC approach leads to accurate results, but becomes computationally costly for highly-collisional cases due to the need of particlevelocity re-sampling at every collision event. On the other hand, one can use a fluid approximation, which can be 
solved in a deterministic way. However, in general, the neutral particles do not reach the fluid limit in every region of the plasma edge.

To increase the accuracy of a fluid model, we presented in Refs. 2.3 a hybrid fluid-kinetic method based on a micro-macro decomposition of the kinetic equation. The micro-macro decomposition 4 leads to a fluid (macro) model with kinetic terms that follow from an MC simulation of a kinetic (micro) correction equation. This micro-macro approach can lead to a significant speed-up compared to the full kinetic MC simulation, depending on the accuracy of the pure fluid solution.

In Refs. 223, we have implemented the hybrid model in a simplified in-house plasma edge code, where we only consider hydrogenic atoms. With the aim of using this hybrid approach for more realistic cases, we present here the integration in the SOLPS-ITER code suite, which consists of the B2.5 plasma fluid finite-volume and EIRENE neutral kinetic MC parts 5. A different hybrid model for the neutrals, which is based on a spatial decomposition in a full kinetic (vacuum regions) and full fluid region (simulated plasma region), is recently implemented in SOLPS-ITER 6. However, this spatially hybrid model does not correct for fluid approximation errors in the simulated plasma domain. Although we consider a case with only deuterium (D), the integration of the hybrid method in SOLPS-ITER enables its use for further multi-species plasma edge simulations.

The paper is outlined as follows. In Section 2, we present the full kinetic equation. The micro-macro decomposition with selected underlying fluid model is given in Section 3 . To facilitate the implementation of the micro part in the extended EIRENE code, there are some crucial differences with the implementation in our in-house code. We pinpoint these differences in Section 4 . In Section 5 , we proceed to the results for a high recycling slab case with fixed background plasma. We compare the sources and we evaluate the performance of the hybrid approach compared to the full kinetic MC simulation in terms of CPU time reduction. We also make a comparison with our in-house code. Finally, Section 6 contains the conclusions and outlook for future work.

\section{$2 \quad$ Full kinetic model}

The (steady-state) kinetic (Boltzmann) equation for the D atoms can be written as

$$
\mathbf{v} \cdot \nabla f_{\mathrm{n}}(\mathbf{r}, \mathbf{v})=S_{\mathrm{c}}(\mathbf{r}, \mathbf{v})+S_{\mathrm{v}}\left(f_{\mathrm{n}}(\mathbf{r}, \mathbf{v})\right)
$$

with $\mathbf{r} \in D \subset R^{2}$ and $\mathbf{v} \in R^{3}$ respectively the position and particle velocity vectors ( $D$ corresponds to the considered plasma edge domain, which is $2 \mathrm{D}$ due to the toroidal symmetry assumption), $\nabla$ the gradient with respect to the position, and $f_{\mathrm{n}}(\mathbf{r}, \mathbf{v})$ the position-velocity phase-space distribution. The right hand side of Eq. (1) contains all kinds of volumetric interactions (sources, sinks, and collisions), with $S_{\mathrm{c}}(\mathbf{r}, \mathbf{v})$ the part independent of $f_{\mathrm{n}}(\mathbf{r}, \mathbf{v})$ and 
$S_{\mathrm{v}}\left(f_{\mathrm{n}}(\mathbf{r}, \mathbf{v})\right)$ the dependent part. We only consider (three-body and radiative) volumetric recombination, electron impact ionization, and charge-exchange $(\mathrm{CX})$ collisions, with respectively rate coefficients $K_{\mathrm{r}}, K_{\mathrm{i}}$, and $K_{\mathrm{cx}}$. Hence, $S_{\mathrm{c}}(\mathbf{r}, \mathbf{v})$ and $S_{\mathrm{v}}\left(f_{\mathrm{n}}(\mathbf{r}, \mathbf{v})\right)$ become

$$
S_{\mathrm{c}}(\mathbf{v})=n_{\mathrm{i}} n_{\mathrm{e}} K_{\mathrm{r}} \widetilde{f}_{\mathrm{i}}(\mathbf{v}), \quad S_{\mathrm{v}}\left(f_{\mathrm{n}}(\mathbf{v})\right)=n_{\mathrm{n}} n_{\mathrm{i}} K_{\mathrm{cx}} \widetilde{f}_{\mathrm{i}}(\mathbf{v})-\left(n_{\mathrm{e}} K_{\mathrm{i}}+n_{\mathrm{i}} K_{\mathrm{cx}}\right) f_{\mathrm{n}}(\mathbf{v})
$$

with $n_{\mathrm{i}}, n_{\mathrm{e}}$ and $n_{\mathrm{n}}=\int_{\mathbf{v}} f_{\mathrm{n}}(\mathbf{v}) \mathrm{d} \mathbf{v}$ respectively the ion, electron, and neutral number densities, with $\int_{\mathbf{v}} \ldots \mathrm{d} \mathbf{v}$ the integral over the whole velocity space $\left(\mathbf{v} \in R^{3}\right)$. The resulting neutral from a recombination or CX event is distributed according to the (normalized) ion distribution $\widetilde{f}_{\mathrm{i}}(\mathbf{v})$, for which we assume a perfect drifting Maxwellian with ion fluid velocity $\mathbf{V}_{\mathrm{i}}$ and temperature $T_{\mathrm{i}}$. In Eq. (2) (and sometimes in upcoming equations), we omit the explicit notation of the position dependency. However, it should be noted that all densities, flow velocities, temperatures, rate coefficients, and velocity distributions depend on $\mathbf{r}$.

At the boundaries of the plasma edge, the incident ions and neutrals are recycled/reflected according to the TRIM database 1 .

\section{Micro-macro model}

For the micro-macro decomposition, we split the total distribution $f_{\mathrm{n}}(\mathbf{v})$ in a fluid (subscript f) and kinetic (subscript k) part, i.e.,

$$
f_{\mathrm{n}}(\mathbf{v})=f_{\mathrm{n}, \mathrm{f}}(\mathbf{v})+f_{\mathrm{n}, \mathrm{k}}(\mathbf{v})
$$

The kinetic part $f_{\mathrm{n}, \mathrm{k}}(\mathbf{v})$ is determined by the micro/kinetic equation, as explained in Section 3.1. The fluid distribution $f_{\mathrm{n}, \mathrm{f}}(\mathbf{v})$ follows from the chosen macro/fluid model, see Section 3.2 .

\subsection{Micro/kinetic part}

Plugging Eq. (3) in Eq. (1) and rearranging the terms leads to a micro equation for $f_{\mathrm{n}, \mathrm{k}}(\mathbf{v})$ :

$$
\mathbf{v} \cdot \nabla f_{\mathrm{n}, \mathrm{k}}(\mathbf{v})=S_{\mathrm{k}, 1}(\mathbf{r}, \mathbf{v})+S_{\mathrm{k}, 2}(\mathbf{r}, \mathbf{v})+S_{\mathrm{v}}\left(f_{\mathrm{n}, \mathrm{k}}(\mathbf{v})\right), \quad \text { with } S_{\mathrm{k}, 1}(\mathbf{r}, \mathbf{v})=S_{\mathrm{c}}, \quad S_{\mathrm{k}, 2}(\mathbf{r}, \mathbf{v})=S_{\mathrm{v}}\left(f_{\mathrm{n}, \mathrm{f}}(\mathbf{v})\right)-\mathbf{v} \cdot \nabla f_{\mathrm{n}, \mathrm{f}}(\mathbf{v}),(4)
$$

where $S_{\mathrm{k}}(\mathbf{r}, \mathbf{v})=S_{\mathrm{k}, 1}(\mathbf{r}, \mathbf{v})+S_{\mathrm{k}, 2}(\mathbf{r}, \mathbf{v})$ corresponds to the (volumetric) source of kinetic neutrals and $S_{\mathrm{v}}\left(f_{\mathrm{n}, \mathrm{k}}(\mathbf{v})\right)$ contains all (volumetric) interactions of the kinetic neutrals, i.e., ionization and CX events. The source $S_{\mathrm{k}}(\mathbf{r}, \mathbf{v})$ can be considered as the residual of Eq. (1) when assuming that $f_{\mathrm{n}, \mathrm{f}}(\mathbf{v})$ is the exact velocity distribution of the total 
population. Instead of using an MC simulation of the full kinetic equation to obtain a certain tally of the full kinetic distribution $f_{\mathrm{n}}(\mathbf{v})$, we perform exclusively an MC simulation for the kinetic correction terms. Special features of the MC procedure are the topic of Section 4

\section{$3.2 \quad$ Macro/fluid part}

The fluid distribution $f_{\mathrm{n}, \mathrm{f}}(\mathbf{v})$ follows from the chosen fluid model. The accuracy of the fluid solution determines the performance of the hybrid method. In SOLPS-ITER, there is already a fluid neutral model available (implemented on the B2.5 side), which consists of a continuity and parallel momentum equation 6 . Now, we add kinetic correction terms to these equations.

A detailed derivation of the macro/fluid equations can be found in Ref. 3. An important feature of the hybrid approach from Ref. 4 is the fact that it is assumed that the macroscopic properties for which we solve fluid moment equations, are entirely determined by the fluid part $f_{\mathrm{n}, \mathrm{f}}(\mathbf{v})$. For the fluid model in SOLPS-ITER, this means that

$$
n_{\mathrm{n}}=\int_{\mathbf{v}} f_{\mathrm{n}}(\mathbf{v}) \mathrm{d} \mathbf{v}=\int_{\mathbf{v}} f_{\mathrm{n}, \mathrm{f}}(\mathbf{v}) \mathrm{d} \mathbf{v}, \quad n_{\mathrm{n}} \mathbf{V}_{\mathrm{n}}=\int_{\mathbf{v}} \mathbf{v} f_{\mathrm{n}}(\mathbf{v}) \mathrm{d} \mathbf{v}=\int_{\mathbf{v}} \mathbf{v} f_{\mathrm{n}, \mathrm{f}}(\mathbf{v}) \mathrm{d} \mathbf{v},
$$

because the zeroth and first order moment equations for respectively particle and momentum balance are solved. Hence, both the kinetic density and kinetic particle flux density become zero, i.e., $\int_{\mathbf{v}} f_{\mathrm{n}, \mathrm{k}}(\mathbf{v}) \mathrm{d} \mathbf{v} \equiv 0$ and $\int_{\mathbf{v}} \mathbf{v} f_{\mathrm{n}, \mathrm{k}}(\mathbf{v}) \mathrm{d} \mathbf{v} \equiv \mathbf{0}$. If a neutral energy equation was included, also the second order moment of $f_{\mathrm{n}}(\mathbf{v})$ would be entirely determined by the fluid part, which means that the kinetic energy density would become zero.

With conditions (5), the continuity and parallel momentum equation become

$$
\nabla \cdot\left(n_{\mathrm{n}} \mathbf{V}_{\mathrm{n}}\right)=-S_{n_{\mathrm{i}}}, \quad \nabla \cdot\left(m n_{\mathrm{n}} u_{\mathrm{n} \| \mid} \mathbf{V}_{\mathrm{n}}-\eta^{\mathrm{n}} \nabla u_{\mathrm{n} \|}+m \int_{\mathbf{v}} \mathbf{v} \mathbf{v} f_{\mathrm{n}, \mathrm{k}}(\mathbf{v}) \mathrm{d} \mathbf{v}\right)=-\nabla_{\|} p_{\mathrm{n}, \mathrm{f}}-S_{m u_{\|}}+S_{\mathrm{cf}},
$$

with $m$ the $\mathrm{D}$ atom particle mass, $u_{\mathrm{n} \|}$ the parallel neutral velocity, $p_{\mathrm{n}, \mathrm{f}}=n_{\mathrm{n}} T_{\mathrm{i}}$ the neutral fluid pressure, and $\eta^{\mathrm{n}}=p_{\mathrm{n}, \mathrm{f}} / n_{\mathrm{i}} K_{\mathrm{cx}}$ the neutral viscosity 7 . With $\nabla_{\|}$, we denote the gradient in the direction parallel to the magnetic field. The plasma particle and parallel momentum source, respectively $S_{n_{\mathrm{i}}}$ and $S_{m u_{\|}}$, are given by

$$
S_{n_{\mathrm{i}}}=-n_{\mathrm{i}} n_{\mathrm{e}} K_{\mathrm{r}}+n_{\mathrm{n}} n_{\mathrm{e}} K_{\mathrm{i}}, \quad S_{m u_{\|}}=-m n_{\mathrm{i}}\left(n_{\mathrm{e}} K_{\mathrm{r}}+n_{\mathrm{n}} K_{\mathrm{cx}}\right) u_{\|}+m n_{\mathrm{n}}\left(n_{\mathrm{e}} K_{\mathrm{i}}+n_{\mathrm{i}} K_{\mathrm{cx}}\right) u_{\mathrm{n} \|},
$$


with $u_{\|}$the plasma parallel velocity. Finally, $S_{\text {cf }}$ partially accounts for the additional terms that arise when taking the parallel component of the vector momentum equation 2. The transport in the perpendicular directions becomes

$$
\left[n_{\mathrm{n}} \mathbf{V}_{\mathrm{n}}\right]_{r, \perp}=-\frac{1}{m\left(n_{\mathrm{i}} K_{\mathrm{cx}}+n_{\mathrm{e}} K_{\mathrm{i}}\right)}\left(\nabla_{r, \perp} p_{\mathrm{n}, \mathrm{f}}+\left[\nabla \cdot m \int_{\mathbf{v}} \mathbf{v v} f_{\mathrm{n}, \mathrm{k}}(\mathbf{v}) \mathrm{d} \mathbf{v}\right]_{r, \perp}\right)
$$

where the subscripts $r$ and $\perp$ are used for respectively the radial and diamagnetic directions.

In contrast to the pure fluid equations, kinetic correction terms appear in Eqs. (6) and (8). This second order moment of the kinetic distribution corrects for the errors from the fluid stress tensor. Including a neutral energy equation would lead to an additional kinetic correction on the fluid heat flux vector.

A similar approach for the boundary conditions, i.e., plugging $f_{\mathrm{n}}(\mathbf{v})=f_{\mathrm{n}, \mathrm{f}}(\mathbf{v})+f_{\mathrm{n}, \mathrm{k}}(\mathbf{v})$ in the full kinetic boundary condition leads to the micro and macro boundary conditions. There is a boundary source for the micro equation, and there are kinetic correction terms in the macro boundary conditions. A detailed elaboration can be found in Ref. 2.

To provide sources of kinetic neutrals in the micro equation (Eq. (4)), we need an expression for the fluid distribution $f_{\mathrm{n}, \mathrm{f}}(\mathbf{v})$. Although $f_{\mathrm{n}, \mathrm{f}}(\mathbf{v})$ is determined by the chosen fluid model, we assume that it is a perfect drifting Maxwellian with the ion temperature, neglecting the neutral perpendicular fluid velocity components. This drifting Maxwellian assumption is the only modeling error (compared to the full kinetic equation) introduced by the micro-macro decomposition. It avoids the need for an extended expression for $f_{\mathrm{n}, \mathrm{f}}(\mathbf{v})$ with, e.g., Hermite polynomials to take into account the viscous contributions and the neglected curvilinear terms. The assumption avoids the implementation of a high number of additional terms in the extended SOLPS-ITER code suite. Hence, deriving and implementing a more correct expression for $f_{\mathrm{n}, \mathrm{f}}(\mathbf{v})$ is kept as future research.

Summarized, we have obtained a coupled set of micro and macro equations, respectively Eq. (4) and Eqs. (6), (8). We use an iterative solution procedure, similar as when solving the coupled fluid plasma-kinetic neutral equations 1. A macro/fluid iteration provides sources for the micro/kinetic equation, and an $\mathrm{MC}$ simulation of the micro equation provides kinetic correction terms for the macro equations. We use the random noise averaging technique from Ref. 88, where we start averaging the outputs of interest over multiple iterations when the transient phase has ended.

\section{Special features of the micro Monte Carlo simulation}

A detailed description of the numerical solution strategy, as implemented in our in-house code, can be found in Ref. 3 . Although the fluid part in B2.5 is similar to that implementation, there are some major differences in the solution algorithm for the micro equation in EIRENE, especially to limit the implementation cost. However, in general, these 
differences lead to an overall decrease in efficiency of the hybrid method. We estimate the performance losses in Section 5.2 In this section, we reveal the main differences between the MC procedure of Ref. 3 and the current paper.

\subsection{Stratified source sampling}

The initial particle position and velocity have to be sampled from the volumetric source distribution $S_{\mathrm{k}}(\mathbf{r}, \mathbf{v})=$ $S_{\mathrm{k}, 1}(\mathbf{r}, \mathbf{v})+S_{\mathrm{k}, 2}(\mathbf{r}, \mathbf{v})$ (defined in Eq. (4)) or boundary source distribution. For the hybrid approach of this paper, both the volumetric and boundary source distributions become negative for some values of $\mathbf{r}$ and $\mathbf{v}$, due to condition (5). In Ref. 3, the positive and negative parts are considered as two independent strata. A particle is launched with an initial weight $w$, where $w$ is positive when the particle originates from the positive stratum, and negative when it originates from the negative stratum.

In the EIRENE code, it is common to define more strata 1, e.g., based on the location of the source (volume, divertor target , ...). Hence, also for this hybrid approach, we subdivide the sources in different strata. The volumetric source $S_{\mathrm{k}}(\mathbf{r}, \mathbf{v})$ consists of three strata: 1) $S_{\mathrm{k}, 1}(\mathbf{r}, \mathbf{v})$ (defined in Eq. (4)), which contains the volumetric recombination; 2) the positive parts of $S_{\mathrm{k}, 2}(\mathbf{r}, \mathbf{v})$ (defined in Eq. (4)); and 3) the negative parts of $S_{\mathrm{k}, 2}(\mathbf{r}, \mathbf{v})$. The volumetric recombination source (1) is already available from the full kinetic simulation. Sampling from $S_{\mathrm{k}, 2}(\mathbf{r}, \mathbf{v})$ will be explained in Section 4.2

Every boundary surface defined in EIRENE consists of three strata: 1) the recycled neutrals (from the surface recombination of incident ions and electrons), which is already present in the full kinetic simulation; 2) the positive part coming from the fluid neutral distribution; and 3) the negative part coming from the fluid neutral distribution. See Ref. 2 for more detailed expressions of the boundary source distributions. We need to sample from a truncated Maxwellian with ion properties for boundary stratum 1, and with fluid neutral properties for boundary strata 2 and 3. The fluid neutral properties are loaded as an additional background in the EIRENE code. We end up with a total of $3 N_{\mathrm{s}}+3$ strata, with $N_{\mathrm{s}}$ the number of boundary surfaces.

\subsection{Sampling from the volumetric source distribution}

In this section, we explain the sampling process for $S_{\mathrm{k}, 2}^{ \pm}(\mathbf{r}, \mathbf{v})$, defined in Eq. (4), with '+' for the positive and '-' for (the magnitude of) the negative stratum. Similar as in Ref. 3 , we make use of the rejection sampling procedure. This means that we sample the initial particle position $\widetilde{\mathbf{r}}$ and velocity $\widetilde{\mathbf{v}}$ from the alternative distribution $S_{\mathrm{k}, 2}^{ \pm *}(\mathbf{r}, \mathbf{v})$. Then, with a probability $S_{\mathrm{k}, 2}^{ \pm}(\widetilde{\mathbf{r}}, \widetilde{\mathbf{v}}) / S_{\mathrm{k}, 2}^{ \pm *}(\widetilde{\mathbf{r}}, \widetilde{\mathbf{v}})$, the position $\widetilde{\mathbf{r}}$ and velocity $\widetilde{\mathbf{v}}$ are accepted, and the particle is launched with initial weight $w= \pm 1$, with ' + ' for the positive and ' - ' for the negative stratum. Otherwise, we sample again from $S_{\mathrm{k}, 2}^{ \pm *}(\mathbf{r}, \mathbf{v})$, until the particle is accepted. 
In Ref. 3 , we subdivide $S_{\mathrm{k}, 2}^{ \pm}(\mathbf{r}, \mathbf{v})$ in different sub-sources to obtain an alternative distribution $S_{\mathrm{k}, 2}^{ \pm *}(\mathbf{r}, \mathbf{v})$ that is close to $S_{\mathrm{k}, 2}^{ \pm}(\mathbf{r}, \mathbf{v})$. This leads to a large acceptance rate, and hence, a low computational cost for the rejection sampling process. However, it is not straightforward to implement the sampling process with $S_{\mathrm{k}, 2}^{ \pm *}(\mathbf{r}, \mathbf{v})$ from Ref. 3 in the extended EIRENE code. Consequently, we take the following alternative distribution functions:

$$
S_{\mathrm{k}, 2}^{ \pm *}(\mathbf{r}, \mathbf{v})=n_{\mathrm{n}} n_{\mathrm{i}} K_{\mathrm{cx}} \tilde{f}_{\mathrm{i}}(\mathbf{v})
$$

which is easier to activate in the EIRENE code. However, one of the the key conditions for the use of rejection sampling is that $S_{\mathrm{k}, 2}^{ \pm *}(\mathbf{r}, \mathbf{v}) \geq S_{\mathrm{k}, 2}^{ \pm}(\mathbf{r}, \mathbf{v})$ for all possible values of $\mathbf{r}$ and $\mathbf{v}$. This condition is violated for the alternative distribution of Eq. (9). Hence, we use following alternative sampling process that combines the rejection and importance sampling procedures:

1. Sample the initial position $\widetilde{\mathbf{r}}$ and $\widetilde{\mathbf{v}}$ from $S_{\mathrm{k}, 2}^{ \pm *}(\mathbf{r}, \mathbf{v})$, given by Eq. (9).

2. If $S_{\mathrm{k}, 2}^{ \pm *}(\widetilde{\mathbf{r}}, \widetilde{\mathbf{v}})<S_{\mathrm{k}, 2}^{ \pm}(\widetilde{\mathbf{r}}, \widetilde{\mathbf{v}})$, then accept the particle and launch it with weight $w= \pm S_{\mathrm{k}, 2}^{ \pm}(\widetilde{\mathbf{r}}, \widetilde{\mathbf{v}}) / S_{\mathrm{k}, 2}^{ \pm *}(\widetilde{\mathbf{r}}, \widetilde{\mathbf{v}})$. If $S_{\mathrm{k}, 2}^{ \pm *}(\widetilde{\mathbf{r}}, \widetilde{\mathbf{v}}) \geq S_{\mathrm{k}, 2}^{ \pm}(\widetilde{\mathbf{r}}, \widetilde{\mathbf{v}})$, then accept the particle with probability $S_{\mathrm{k}, 2}^{ \pm}(\widetilde{\mathbf{r}}, \widetilde{\mathbf{v}}) / S_{\mathrm{k}, 2}^{ \pm *}(\widetilde{\mathbf{r}}, \widetilde{\mathbf{v}})$, otherwise go back to step 1.

This combined rejection-importance sampling process makes sure that we sample from the exact $S_{\mathrm{k}, 2}^{ \pm}(\mathbf{r}, \mathbf{v})$, and limits the variance of the output tallies. However, the acceptance rate (and hence the performance) is reduced compared to Ref. 3 .

\subsection{Simulating the particle trajectories}

Typically, EIRENE uses an analog simulation for volumetric processes. This means that the particle weight is kept constant during the trajectory in the plasma edge volume. Only at a boundary, the particle weight is reduced if the recycling/reflection coefficient is smaller than one. At a collision event, the particle trajectory is ended with probability $R_{\mathrm{a}} / R_{\mathrm{t}}$, with $R_{\mathrm{a}}$ and $R_{\mathrm{t}}$ respectively the local absorption and total reaction rates. For the full kinetic simulation here, $R_{\mathrm{a}}=n_{\mathrm{e}} K_{\mathrm{i}}$ and $R_{\mathrm{t}}=n_{\mathrm{e}} K_{\mathrm{i}}+n_{\mathrm{i}} K_{\mathrm{cx}}$. The tallies are calculated with track-length estimators 9 , where the tallies are updated on the particle trajectory between collision events. The analog simulation in combination with track-length estimators performs well for CX dominated cases 9 .

For the hybrid model, $R_{\mathrm{a}}=R_{\mathrm{t}}=n_{\mathrm{e}} K_{\mathrm{i}}+n_{\mathrm{i}} K_{\mathrm{cx}}$. This means that at every collision event, the particle is immediately absorbed. Hence, when the full kinetic simulation is CX dominated, the hybrid micro simulation becomes absorption dominated. According to Ref. 9, the analog simulation in combination with a non-analog track-length estimator is far from optimal w.r.t. the statistical error for a certain CPU cost for absorption-dominated cases. Due to the 
different character of the hybrid micro equation compared to the full kinetic equation, it is recommended to, e.g., use a non-analog track-length type simulation in combination with the track-length estimator. However, improving the simulation and estimator types is kept for future research.

\section{Results}

We assess the hybrid model for a slab case with a fixed background plasma that is typical for a high recycling case. Fig. Ia) shows a poloidal cross-section of the slab geometry with the different regions (core, private flux (PF), and scrape-off layer (SOL)). The cuts (in pink) represent a kind of artificial distinction between the core and PF regions, where all particles hitting a cut (on one of both sides) are specularly reflected. The total amount of MC particles is distributed over the strata according to their source strengths (based on the pure fluid solution for the hybrid simulation). A detailed description of the case with plots of the background plasma can be found in Ref. 2 . In Section 5.1, we compare the kinetic, fluid, and hybrid plasma sources. In Section 5.2, we compare the needed CPU time for the kinetic and hybrid simulations for a certain statistical error.

\subsection{Plasma sources}

In Figs. 1 (b)-(e), we compare the resulting plasma sources in the vicinity of a divertor target from the pure kinetic, pure fluid, and hybrid fluid-kinetic simulations in three relevant flux tubes, which are located in the proximity of the separatrix $(R=2.54 \mathrm{~m})$, with $Z_{\mathrm{t}}$ the distance from the target. To not overload the figures, we only show the results for the red flux tube in Figs. 1(nc)-(d). The results in the other flux tubes are qualitatively the same. The hybrid approach significantly reduces the differences with the kinetic solution. However, there are some remaining hybrid-kinetic discrepancies due to numerical errors (discretization error and bias) and the modeling error in the assumption of a perfect drifting Maxwellian neutral equilibrium distribution (see Section 3.2. In Ref. 2, we show that the modeling error is dominant for this specific case.

\subsection{Reduction of CPU time}

We assess the performance of the hybrid method based on the reduction of the CPU time compared to a full kinetic MC simulation to obtain the same statistical error on a certain source. The statistical error is defined as the L2-norm over all grid cells. For a full kinetic MC simulation, the statistical error roughly scales with $1 / \sqrt{C P U}$, with $C P U$ the CPU time. This assumption is valid due to the fact that the CPU time is approximately proportional to the number of MC particles. The $1 / \sqrt{C P U}$ scaling still holds for the hybrid simulation, because we average the solution over multiple iterations 8 and the computational cost for the transient phase is negligible for this fixed background plasma simuation. 


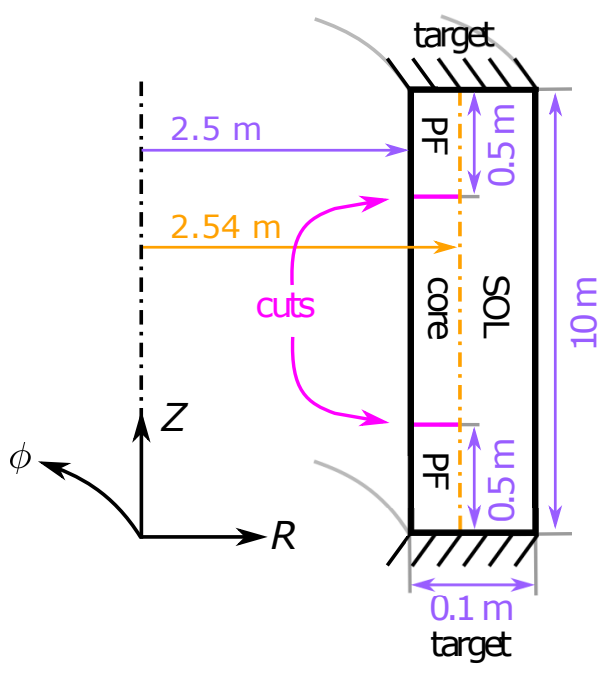

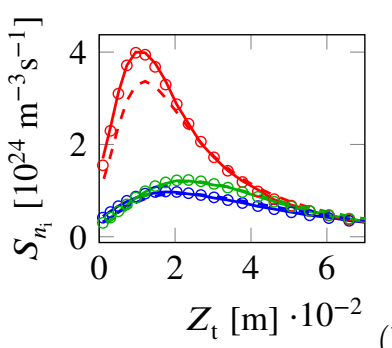
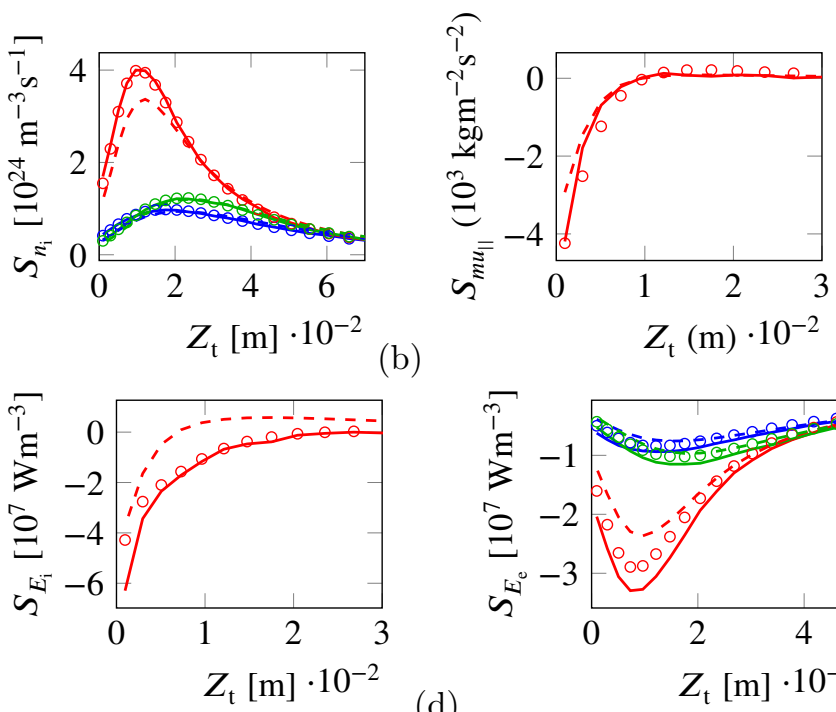

(b)

(b)

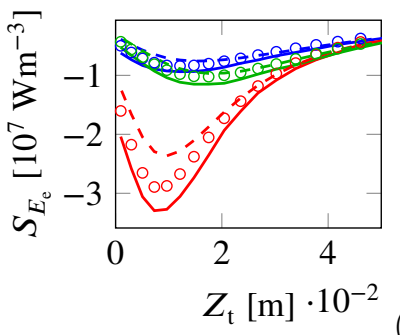

(d)

Figure 1: Poloidal cross-section of the slab geometry (a) and plasma sources in selected flux tubes at $R=2.53 \mathrm{~m}$ (blue), $R=2.55 \mathrm{~m}$ (red), and $R=2.56 \mathrm{~m}$ (green) (b-e): kinetic MC (solid lines), fluid (dashed lines) and hybrid (circular marks) solutions. (a) Particle $\left(S_{n_{\mathrm{i}}}\right)$, (b) parallel momentum $\left(S_{m u_{\mid l}}\right)$, (c) (total) ion energy $\left(S_{E_{\mathrm{i}}}\right)$, and (d) electron energy $\left(S_{E_{\mathrm{e}}}\right)$ source.

\begin{tabular}{l|cccc}
\hline & $\epsilon_{S_{n_{\mathrm{i}}}}$ & $\epsilon_{S_{m u_{\|}}}$ & $\epsilon_{S_{E_{\mathrm{i}}}}$ & $\epsilon_{S_{E_{\mathrm{e}}}}$ \\
\hline SOLPS-ITER & 1.73 & 4.87 & 0.29 & 1.91 \\
In-house code & 3.53 & 10.56 & 0.90 & 3.54 \\
\hline
\end{tabular}

Table 1: CPU time reduction of the hybrid approach compared to the full kinetic MC simulation to obtain the same statistical error on a certain source.

Table 1 Hhows the CPU time reduction $\epsilon_{S}$ for the same statistical error on a certain source $S$. If $\epsilon_{S}>1$, then the hybrid model is $\epsilon_{S}$ times faster, otherwise, it is $1 / \epsilon_{S}$ times slower. Due to the $1 / \sqrt{C P U}$ scaling for both full kinetic and hybrid simulations, $\epsilon_{S}$ does not depend on the CPU time. The table also contains the speed-up factors obtained with our in-house code, as reported in Ref. 2. We conclude that the hybrid approach with this specific underlying fluid model reduces the CPU time for all sources, except for the ion energy source. However, there is less CPU time reduction in SOLPS-ITER than in our in-house code. We expect that the performance losses are mainly caused by the analog particle trajectory simulation strategy, as explained in Section 4.3 .

Due to conditions (5), there are no direct kinetic contributions in $S_{n_{\mathrm{i}}}, S_{m u_{\|}}$, and $S_{E_{\mathrm{e}}}$. However, the nonzero second order moment of $f_{\mathrm{n}, \mathrm{k}}(\mathbf{v})$ directly contributes to the ion energy source deteriorating the statistics of $S_{E_{\mathrm{i}}}$. This explains the CPU time increase for $S_{E_{\mathrm{i}}}$ in Table 1 An increased performance for the ion energy source can be obtained by solving a separate neutral energy equation, as shown in Ref. 3 . 


\section{Conclusions and future work}

We conclude that the hybrid approach based on a micro-macro decomposition of the kinetic equation is able to significantly reduce the fluid-kinetic discrepancies. There is a speed-up compared to the full kinetic MC simulation to obtain the same statistical error on the particle, momentum, and electron energy source. Unfortunately, there is no speed-up for the ion energy source. As shown in Ref. 3, the performance can still be increased by adding a separate neutral energy equation with also a CPU time reduction for the ion energy source. Implementation of such a separate neutral energy equation into SOLPS-ITER is currently ongoing, with promising first results 10 . The performance of the hybrid model in SOLPS-ITER has decreased compared to our in-house code due to some nonoptimal implementation decisions for the ease of integration of the micro part in the extended EIRENE code. It is recommended to update the particle trajectory simulation strategy and possibly the estimators.

In future research, the coupled fluid plasma-hybrid neutral equations should be solved to assess the impact of the individual source variance reductions (or increase for the ion energy source) on the plasma-dependent outputs of interest. This hybrid method is expected to become especially advantageous in (partially) detached regimes, where charge-exchange collisions can significantly increase the computational cost when using a full kinetic Monte Carlo approach for the neutrals. In addition, nonlinear neutral-neutral collisions can become important in detached divertor conditions 11. Although the hybrid approach simplifies the treatment of neutral-neutral collisions, extending the hybrid model for neutral-neutral collisions is kept as future research.

\section{Acknowledgments}

The work of N. Horsten is supported by a PhD grant of the Research Foundation Flanders (FWO Vlaanderen). M. Blommaert is a postdoctoral research fellow of the FWO and the Flemish Institute for Technological Research (VITO). Parts of the work are supported by the FWO project with grant number G078316N. The computational resources and services used in this work were provided by the VSC (Flemish Supercomputer Center), funded by the FWO and the Flemish Government - department EWI.

\section{References}

[1] D. Reiter, M. Baelmans, P. Börner, Fusion Science and Technology 2005, 47 (2), 172-186.

[2] N. Horsten, G. Samaey, M. Baelmans, Nuclear Materials and Energy 2019, 18, 201-207.

[3] N. Horsten, G. Samaey, M. Baelmans, Journal of Computational Physics, in press.

[4] A. Crestetto, N. Crouseilles, M. Lemou, Kinetic and Related Models 2012, 5 (4), 787-816. 
[5] S. Wiesen, D. Reiter, V. Kotov, M. Baelmans, W. Dekeyser, A.S. Kukushkin, S.W. Lisgo, R.A. Pitts, V. Rozhansky, G. Saibene, et al., Journal of Nuclear Materials 2015, 463, 480-484.

[6] M. Blommaert, N. Horsten, P. Börner, W. Dekeyser, Nuclear Materials and Energy 2019, 19, 28-33.

[7] N. Horsten, G. Samaey, M. Baelmans, Nuclear Fusion 2017, 57 (11), 116043.

[8] K. Ghoos, W. Dekeyser, G. Samaey, P. Börner, M. Baelmans, Journal of Computational Physics 2016, 322 (C), $162-182$.

[9] B. Mortier, M. Baelmans, G. Samaey, submitted to Computer Methods in Applied Mechanics and Engineering.

[10] W. Van Uytven, M. Blommaert, W. Dekeyser, N. Horsten, M. Baelmans, submitted to Contributions to Plasma Physics.

[11] D. Reiter, V. Kotov, P. Börner, K. Sawada, R.K. Janev, B. Küppers, Journal of Nuclear Materials 2007, 363, $649-657$. 
How cite this article: N. Horsten, W. Dekeyser, M. Blommaert, G. Samaey, and M. Baelmans (2019), A hybrid fluid-kinetic neutral model based on a micro-macro decomposition in the SOLPS-ITER plasma edge code suite, Contributions to Plasma Physics, . 